\title{
Clinical applications of laser speckle contrast imaging: a review
}

\author{
Wido Heeman \\ Wiendelt Steenbergen \\ Gooitzen M. van Dam \\ E. Christiaan Boerma
}




\title{
Clinical applications of laser speckle contrast imaging: a review
}

\author{
Wido Heeman, ${ }^{a, b, c}$ Wiendelt Steenbergen, ${ }^{d}$ Gooitzen M. van Dam, ${ }^{b}$ and E. Christiaan Boerma ${ }^{e, *}$ \\ aUniversity of Groningen, Faculty Campus Fryslân, Leeuwarden, The Netherlands \\ bUniversity Medical Centre Groningen, Department of Surgery, Optical Molecular Imaging Groningen, Groningen, The Netherlands \\ 'LIMIS Development BV, Leeuwarden, The Netherlands \\ dUniversity of Twente, Techmed Center, Faculty of Science and Technology, Biomedical Photonic Imaging Group, Enschede, The Netherlands \\ ${ }^{\mathrm{e}}$ Medical Centre Leeuwarden, Department of Intensive Care, Leeuwarden, The Netherlands
}

\begin{abstract}
When a biological tissue is illuminated with coherent light, an interference pattern will be formed at the detector, the so-called speckle pattern. Laser speckle contrast imaging (LSCI) is a technique based on the dynamic change in this backscattered light as a result of interaction with red blood cells. It can be used to visualize perfusion in various tissues and, even though this technique has been extensively described in the literature, the actual clinical implementation lags behind. We provide an overview of LSCI as a tool to image tissue perfusion. We present a brief introduction to the theory, review clinical studies from various medical fields, and discuss current limitations impeding clinical acceptance. $\odot$ The Authors. Published by SPIE under a Creative Commons Attribution 4.0 Unported License. Distribution or reproduction of this work in whole or in part requires full attribution of the original publication, including its DOI. [DOI: 10.1117/1.JBO.24.8.080901]
\end{abstract}

Keywords: laser speckle contrast imaging; burn wounds; retinal perfusion; cerebral blood flow; microcirculation.

Paper 190122VRR received Apr. 15, 2019; accepted for publication Jul. 2, 2019; published online Aug. 5, 2019.

\section{Introduction}

Perfusion is an indicator for tissue viability, and several laserbased optical imaging modalities to assess this have been developed. Medical users want a noncontact, fast, simple, and cheap modality. ${ }^{1}$ Laser speckle contrast imaging (LSCI) is a fast, full-field, cheap, and relatively simple imaging method that can give 2-D perfusion maps of large surfaces. ${ }^{2}$ LSCI is based on the principle that the backscattered light from a tissue that is illuminated with coherent laser light forms a random interference pattern at the detector, the so-called speckle pattern. Movement of particles inside the tissue causes fluctuations in this speckle pattern resulting in blurring of speckle images when obtained with an exposure time equal to or longer than the speckle fluctuation time scale. This blurring can be related to blood flow if the fluctuations are caused by the movement of red blood cells (RBCs). Starting out as a slow analogue research tool, LSCI systems can now image blood flow in (near) realtime as a result of the rapid increase in cheap computing power. This empowers the translation of LSCI into clinical practice where it is well suited for the assessment of perfusion in a wide range of tissues. LSCI has been applied to image burn wounds, retinal perfusion, cerebral blood flow $(\mathrm{CBF})$, skin microvasculature, liver, esophagus, and the large intestine. Despite the vast amount of research on LSCI, few applications are commonly applied in clinical practice. This review serves as an introductory overview of current clinical applications of LSCI for medical specialists.

\section{Laser Speckle Contrast Imaging}

The first biomedical application was reported by Fercher and Biers. $^{2}$ The technique proposed by Fercher and Briers was non-real-time and had practical limitations due to the use of

*Address all correspondence to E. Christiaan Boerma, E-mail: e.boerma@ chello.nl nondigital systems, which impeded the clinical use. The first real speed increases to quasi real-time image acquisition and processing happened in the $1990 \mathrm{~s}^{3}$ with the introduction of digital photography. ${ }^{4}$ As mentioned previously, most of the essential developments in the applicability of LSCI as a clinical tool took place in the last 15 years with the vast increase in cheap computer power. For instance, Richards et al. ${ }^{5}$ were able to build an LSCI setup for 90 USD with an imaging performance comparable to a 2000 USD setup. Generally, the components required are a low-powered laser diode, a diffuser, a digital camera, and processing software (Fig. 1). Currently, there are at least two companies that have commercialized LSCI. Other names for the same principle as LSCI are laser speckle imaging (LSI), laser speckle perfusion imaging, and laser speckle contrast analysis (LASCA) as it was named by the first users.

\subsection{Basic Principles of Laser Speckle Contrast Imaging}

Speckle patterns are the random interference patterns that arise when coherent light is backscattered by a scattering medium such as biological tissue. The slightly different optical pathlengths cause the waves to reach the observer at random mutual phases, resulting in bright and dark spots, respectively. ${ }^{6}$ The speckle image is built up of static and dynamic speckles. Static speckles are speckles that do not change over time, whereas dynamic speckles do change over time due to the optical Doppler effect. The dynamic speckles contain information about movement of the object or motion of particles within the object.

In order to be able to detect a change in the speckle pattern, the exposure time of the camera must be of the order of the speckle decorrelation time, causing a blurring of the recorded speckle pattern. It is this blurring that is used to calculate the speckle contrast $K$ using the following formula:

$$
K=\frac{\sigma}{\langle I\rangle}
$$




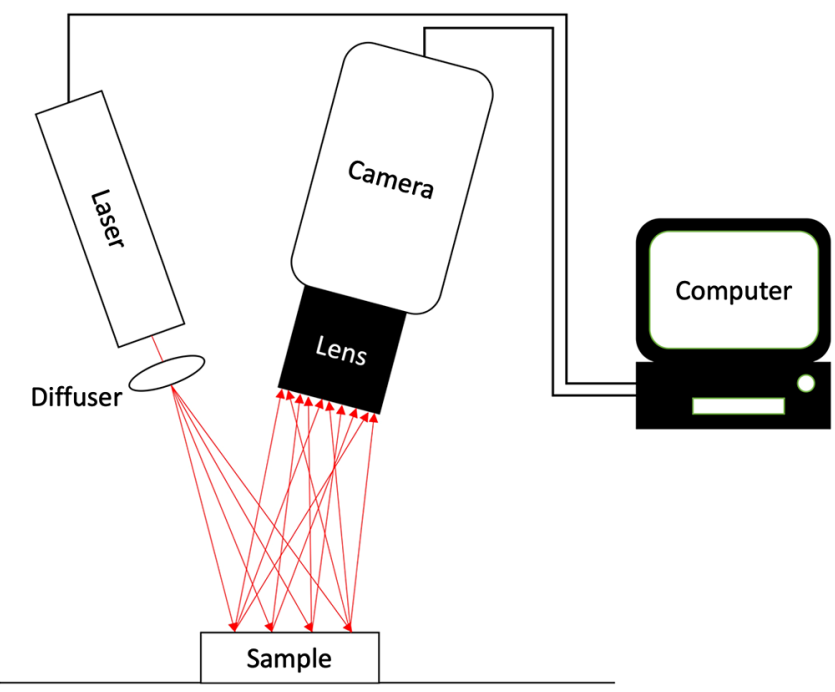

Fig. 1 A typical LSCl setup with a laser, diffuser, camera, and processing software.

where $\sigma$ is the standard deviation of the intensity $I$ over the mean intensity $\langle I\rangle$ calculated over a window in space or time. Spatial contrast uses an area of multiple pixels in one frame, as can be seen in the bottom left corner of Fig. 2. A window size of $5 \times 5$ or $7 \times 7$ pixels has been suggested for optimal results. ${ }^{7}$ Spatial contrast decreases the spatial resolution; however, this method does have a high temporal resolution. Temporal LSCI uses the same pixel in multiple frames to calculate the contrast in a time window, as can be seen in the top right corner of Fig. 2. Temporal contrast has a high spatial resolution and low temporal resolution. Spatial contrast has superior temporal resolution and vice versa ${ }^{8}$ hence, it can be beneficial if combined into a so-called spatio-temporal contrast as can be seen in the top

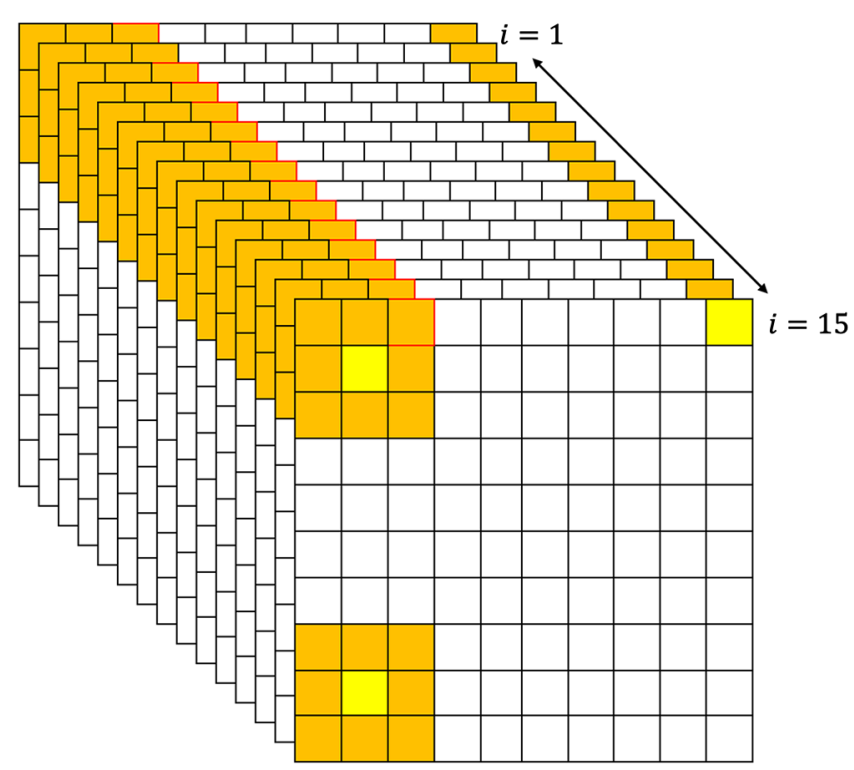

Fig. 2 A pixel matrix where the orange pixels are used to calculate the contrast for the yellow pixel. In the top right corner, a graphical representation of temporal contrast calculated over 15 frames. In the bottom left corner, a representation of spatial contrast assuming a $3 \times 3$ window. In the top left corner, a representation of spatiotemporal contrast calculated over 15 frames. left corner of Fig. 2. The choice of resolution should be based on the need for a high temporal or spatial resolution.

If the exposure time of the detector is infinitesimally small or shorter than the intensity fluctuation time of the speckles, the standard deviation $\sigma$ is equal to the mean intensity $\langle I\rangle$, which theoretically results in a contrast value of $K=1$. If there is movement present and the exposure time of the detector is of the order of or longer than the fluctuation time, the picture will be blurred meaning that the standard deviation $\sigma$ will be small compared to the mean intensity $\langle I\rangle$, which results in a loss of contrast, hence, $0 \geq K<1$.

\subsection{Multi-Exposure Speckle Imaging}

As mentioned above, the amount of blurring is dependent on the movement of particles within the object and the exposure time. The first applications of LSCI were single-exposure methods, meaning that the exposure time is kept constant with every measurement. Single exposure has the disadvantage that it is hard to quantify the measured perfusion since the sensitivity and quantitative accuracy are highly dependent on the exposure time. ${ }^{9}$ In an attempt to make the method more robust and to increase the reproducibility of results, Parthasarathy et al. ${ }^{10}$ developed the first multi-exposure laser speckle imaging (MESI) setup. This new setup can vary the exposure time while maintaining a constant intensity. In addition to the multi-exposure setup they also derived a new, more complete, mathematical model for speckle imaging by considering the presence of static scattered light. The multi-exposure setup shows linearity with relative changes in speed over a broader range of velocities, whereas the single-exposure setup is becoming less accurate for larger variations. This allows for semiquantitative measurements. The proposed speckle model can be used to obtain the systems noise, the fraction of dynamically scattered light $\rho$, and the correlation time $\tau_{c}$ based on the Lorentzian velocity distribution. $^{10}$

\subsection{Velocity Distributions}

For the calculated contrast $K$ [Eq. (1)] to be linked to velocity and the RBC concentration, the velocity distribution must be known. ${ }^{11}$ This relation is described by Eq. (2) for a Lorentzian velocity distribution. ${ }^{12}$ In this equation, the speckle contrast $K$ is dependent on exposure time $T$ and speckle correlation time $\tau_{c}$, where $K, T$, and $\tau_{c}$ are linked via the theory of correlation functions and time integrated speckle. By doing so, one can calculate $\tau_{c}$, and the subsequent estimate of RBC velocity using the approximation $\tau_{c} \propto \frac{1}{V}$, where $V$ is velocity. ${ }^{10}$ Fercher and Briers chose to use the Lorentzian distribution; however, an alternative interpretation of velocity distribution is suggested by others. ${ }^{7,13,14}$ The Lorentzian velocity distribution is valid for Brownian movement or unordered flow, which is not conclusive in a clinical setting. The Gaussian velocity distribution is a better fit for ordered flow. Notwithstanding, it has become clear that the true velocity distribution is more complex than purely Lorentzian or Gaussian. Smausz et al. ${ }^{15}$ reverse engineered some extra parameter $\alpha$ with which they multiplied $T / \tau_{c}$ to get a better fitting to the theoretical contrast curve using multiple exposures ranging from 0.2 to $500 \mathrm{~ms}$ as in Eq. (3). This parameter was used to compensate for the static speckles and could also be used in single-exposure LSCI after determination using MESI. Yet, these results require further research due to the seemingly incorrect use of the Lorentzian velocity distribution. Duncan and 


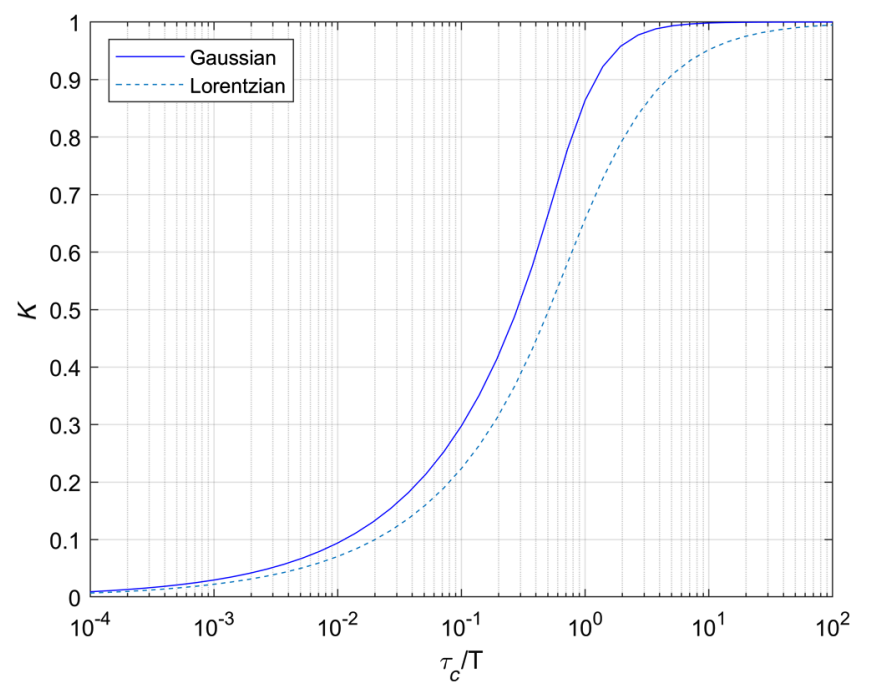

Fig. $3 \mathrm{~A}$ graph displaying the relation between the speckle contrast and the speckle decorrelation time $\tau_{c}$ over the integration time $T$ for the Lorentzian (right) and Gaussian (left) velocity distributions.

Kirkpatrick $^{13}$ also pointed out that the characteristic correlation times $\tau_{c}$ are dependent on a combination of ordered flow and unordered flow. The actual correlation time is somewhere between the two distributions with the Gaussian and Lorentzian as limiting behaviors as in Fig. 3 .

Smausz et al. ${ }^{15}$ argued that a correlation law based on the rigid-body motion could be the answer. In fact, the rigid-body model is a hybrid between Gaussian and Lorentzian with the prevalent behaviors at short and long exposures, respectively. A recurring problem in literature is that the incorrect velocity distribution is often used resulting in a flawed correlation function. Next to that, multiple groups have confirmed that the contrast equation should consider the effect of triangular averaging: ${ }^{8,13,15-18}$

$K(T)=\beta^{0.5}\left(\frac{\tau_{c}}{2 T}+\frac{\tau_{c}^{2}}{2 T^{2}}\left\{\left[1-\exp \left(-\frac{2 T}{\tau_{c}}\right)-1\right]\right\}\right)^{0.5}$

$K(T)=\alpha \frac{T}{\tau_{c}}$

\subsection{Does Laser Speckle Contrast Imaging Measure Flow or Velocity?}

First, to elucidate the appropriate definitions; flow is defined as a volume per time unit, whereas velocity is defined as distance per time unit. It is accepted that laser Doppler flowmetry (LDF) measures flow. ${ }^{7,14}$ LSCI is in some aspects similar to laser Doppler although it still lacks this level of acceptance in this field. It can be stated that it is still indeterminate whether LSCI measures velocity or flow. In a situation where there is no movement of RBCs, only static speckles are present with no blurring and theoretically a contrast of $K=1$. Consider the fact that a fraction of the RBCs starts to move, which leads to Doppler shifted photons. A fraction of the static speckles has turned into dynamic speckles decreasing the contrast $K$. However, it is unclear if the origin of the decrease in speckle contrast, and the corresponding $\tau_{c}$, is caused by an increase in flow or velocity. Yet, it is clear that this must be related to the RBCs movement within the exposure time. Eq. (2) relates contrast $K$ to the correlation time $\tau_{c}$, which is inversely proportional to velocity. ${ }^{7}$ The loss in contrast is directly related to the concentration and size of scatterers in the measured medium as said by Nadort et al. ${ }^{19}$ This group studied the influence of velocity, scatterer size, and concentration on the measured contrast $K$ in a flow phantom. An analysis is made with differences in flow speed, scatterer size, and concentration that all influence the contrast $K$ and thus the relation $1 / \tau_{c}=\alpha V$. Different velocities for different scatterer size and scatterer concentration all give slightly different contrast values for different exposure times. Finally, it is generally accepted that the loss in contrast is caused by the movement of RBCs, although the relation is not unanimously accepted yet.

\subsection{Movement Artefact Correction}

LSCI is extremely sensitive to motion. It is able to detect the small-scale movements of RBCs; however, this is the power and pitfall of this technology. ${ }^{20}$ Unwanted movement artifacts are embodied in the measured signal and cannot be easily separated. A simple movement artefact precaution could involve establishing a distraction free and quiet environment for the patient. ${ }^{21}$ Mahé et al. ${ }^{22}$ added an adhesive opaque patch within the field of view. This is based on the idea that the opaque surface speckle contrast contains solely information about unwanted movement and not perfusion. The measured contrast on the exposed skin contains information about perfusion and unwanted movement; as such when the opaque surface contrast is deducted, with the addition of a linearity correction, only perfusion is left. This method was later used by others. ${ }^{23-25}$ The same group later improved this method with an equation that is valid for the studied population that does not require calibration. ${ }^{26}$ Using this method, cutaneous microvascular assessment was measured on a patient forearm during exercise. ${ }^{27}$ The forearm was placed on a table while the patient was working out on a home trainer. Whereas Mahe et al. used the adhesive opaque patch as a zeroflow reference, and Lertsakdadet et al. ${ }^{25}$ used a fiducial marker to identify and re-align a subset of speckle images that have an acceptable degree of motion artefact. These methods can be helpful when cutaneous microvascular perfusion is assessed, yet in some cases the tissue of interest cannot have any object stuck to it. For example, Richards et al. ${ }^{28}$ applied an electrocardiogram (ECG)-based post hoc correction on CBF measurements to correct for the palpable motion of the heartbeat. Finally, Miao et al. ${ }^{29}$ proposed registered laser speckle contrast analysis based on a modified registration technique, to post hoc compensate for respiration and heart beating in animals. The need for a robust, real-time, yet effective motion correction still exists. ${ }^{7}$ This can then be applied in any situation such as intra-abdominal cavities and $\mathrm{CBF}$ measurements.

\section{Clinical Applications of Laser Speckle Contrast Imaging}

This review is limited to preclinical and clinical LSCI literature. The discussed research fields are chosen based on the authors' interests.

\subsection{Rheumatology}

Perfusion assessment using LSCI is applied in rheumatology to determine the state of systemic sclerosis (SSc). A study by Della Rossa et al. ${ }^{30}$ was the first to report on the use of LSCI to monitor the dynamic vascular reactivity and blood flow in patients 
affected by Raynaud's phenomenon including SSc patients. A differentiation between SSc and primary Raynaud's phenomenon could be made solely based on qualitative blood perfusion measures such as peak flow after an ischemic test and postischemic hyperemic area under the curve. This was later verified by others. ${ }^{31}$ Ruaro et al. ${ }^{32}$ went one step further by verifying the LSCI data with LDF and nailfold video capillaroscopy (NVC). LSCI and LDF were used to measure the peripheral blood perfusion (PBP). NVC is the standard of care in rheumatology to determine the state of SSc (early, active, or late) by providing information on the morphological microvascular abnormalities of the capillaries. The PBP values measured using LSCI and LDF were found to have a linear relationship between different NVC patterns (Fig. 4). Lower PBP values were found for SSc patients compared to healthy subjects, which is in line with findings of others ${ }^{33}$ also using $\operatorname{LDF}^{34,35}$ and laser Doppler imaging (LDI). ${ }^{36}$ In contrast to a previous publication, ${ }^{37}$ Wilkinson et al. ${ }^{38}$ reported good potential in comparison to thermography. With these results considered, LSCI is a reliable technique for detecting PBP in humans. The PBP values correlate well with the progression of SSc and NVC patterns. ${ }^{39}$ LSCI is

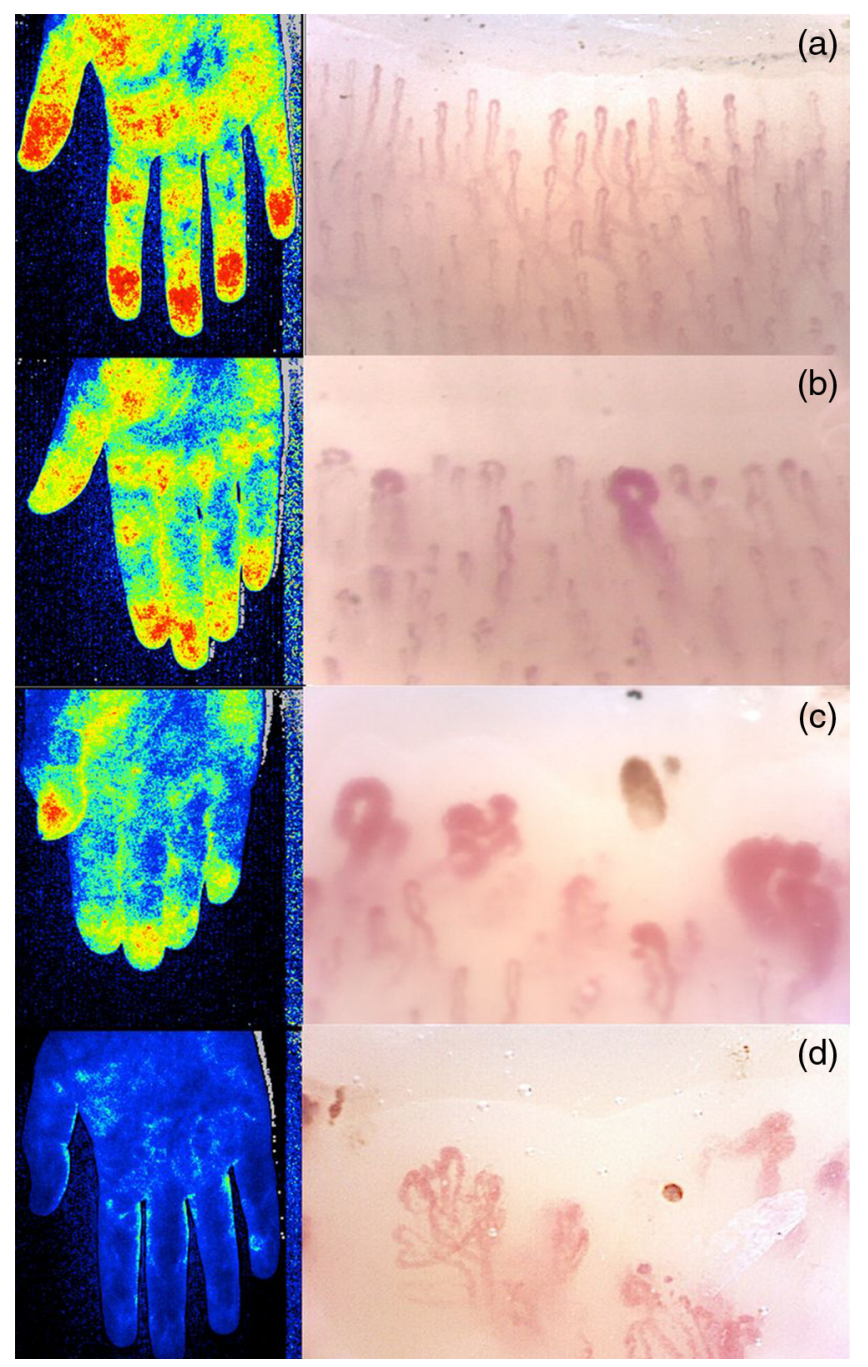

Fig. 4 LASCA image of right hands and related NVC pictures in (a) a healthy subject, and in SSC patients with (b) "early, (c) "active", or (d) "late" pattern of microangiopathy (NVC picture magnification 200x). Figure reprinted from Fig. 1 in Ref. 32, ( 2019, with permission from BMJ. still a relatively new technique in this field, however, it gains popularity over the more widely accepted LDF because of its shorter acquisition time, higher reproducibility because of decreased intraoperator variability, ease of use due to the elimination of the need of a full-contact probe,,$^{30,32}$ and the fact that LSCI provides an image rather than a single-point measurement.

A more prevalent disease in rheumatology is arthritis. Arthritis is caused by an inflammatory reaction affecting joints that can be characterized by locally increased perfusion. Different stages of arthritis can already be successfully characterized by LSCI in animal models. ${ }^{40}$ Detection in early stages of arthritis might help to delay or prevent further damage if the characteristic neo-angiogenesis in humans can be quantified using LSCI. This study was performed in a mouse model and to date no human in vivo studies have been performed. ${ }^{40}$ This could be related to the inadequate superficial measuring depth of a standard LSCI setup. Dunn et al. ${ }^{41}$ showed that by using their so-called transmissive LSI technique, this depth can be increased to 10 to $15 \mathrm{~mm}$ into tissues, which is sufficient to monitor finger joint synovial blood flow. The transmissive laser speckle imager is an instrument where the laser source is opposed to the detector instead of alongside. Finally, Forrester et al. ${ }^{42}$ reported on a new endoscopic apparatus that was used to image the rabbit joint capsule tissue in vivo. The same group also reported on the successful application of the same apparatus in the human knee of patients requiring arthroscopic knee surgery. ${ }^{43}$ Many diseases in rheumatology are characterized by a change in microvascular perfusion, which implies that LSCI could become an important tool for the rheumatologist for evaluation of disease activity. Yet, the inadequate penetration depth to reach the tissue of interest hinders full clinical acceptation and application broader than characterization of SSc patients.

\subsection{Burns}

Unlike the rheumatologist, a burn surgeon is in most instances working with superficial tissues such as the skin or superficial muscle tissue. An example is burn wound assessment. The clinical observation based on visual and tactile information of burn wound severity by skilled burn surgeons only suffices in about $70 \%$ to $80 \%$ of the time. ${ }^{44}$ The two extremes, red painful, nonblistering superficial burns and pale, leathery insensate deep burns, are easy to detect for most clinicians. It is much harder to determine correct severity of the partial-thickness burns that greatly influences the treatment. ${ }^{45}$ An incorrect diagnosis of deep partial-thickness wounds can lead to unnecessary hospitalization, excision, and grafting. However, the incorrect diagnosis of superficial partial-thickness wounds can lead to sepsis-related morbidity. ${ }^{46}$ Hence, the clinical need for a robust objective method is critical. The state of microcirculatory blood flow is an indicator of healing capability and thus imaging modalities able to visualize the blood flow can be of great help to the burn surgeon. Currently, LDI is the most used clinically although it has its drawbacks of being costly, bulky, and having long acquisition times. ${ }^{47}$ LSCI, on the other hand, can achieve similar results while sustaining the advantage of being noninvasive and noncontact with the added advantage of being cheap, simple, and having short acquisition times. ${ }^{48}$ The latter is important for treating patients who cannot remain still because they are in pain or because it concerns infants.

Stewart et al. ${ }^{48}$ were the first to report on the use of LSCI to asses superficial blood flow within burn surgery. They measured 
burn scar perfusion and made a comparison between LDI and LSCI concluding with a favorable note toward LSCI. Crouzet et al. ${ }^{49}$ used LSCI to measure perfusion in a mouse burn model. They induced both superficial-partial and deep-partial thickness burns. Results indicate that LSCI can discriminate between the induced superficial-partial and deep-partial burns with statistical significance. These results are very promising even though a translation to clinically relevant data could be complex due to the inevitable biological differences in skin such as thickness and structure. Lindahl et al. ${ }^{50}$ did a study using LSCI on pediatric scald injuries trying to predict the outcome of the burn at 14 days postburn. LSCI is well suited for pediatric burns due to the short acquisition time. Their results show a significant difference after one day for wounds that healed within 14 days compared with those who underwent surgery. Further validation of various burn types required for clinical application was later done by the same group. ${ }^{51,52}$ LSCI is of help with easy to understand images that can predict wound healing (Fig. 5). A singleperfusion measurement is sufficient to predict the need for surgery with increased accuracy when an additional measurement is made between 0 and $24 \mathrm{~h}$ after burn. LSCI should be used together with the surgeon's expertise since perfusion measurements do not rule out deep or superficial burns. However, it can be a valuable tool for the surgeon for clinical decisionmaking in superficial wounds for the need of surgery.

\subsection{Dermatology}

LSCI is used within dermatology for monitoring port wine stain (PWS) birthmarks. PWS are progressive vascular malfunctions with locally increased blood flow. PWS birthmarks are commonly treated using a pulsed dye laser that causes vascular damage. ${ }^{53}$ Huang et al. ${ }^{54,55}$ were the first to monitor the effect of pulsed dye laser treatment on the perfusion. They found that the perfusion was significantly decreased in treated areas, whereas the untreated areas remained unchanged. This suggests that LSCI is a potential surgical guidance tool that can decrease the amount of sessions required for complete PWS blanching. ${ }^{56,57}$ The effect of PWS blanching is clearly presented in Fig. 6. Next to this, Yang et al. ${ }^{58}$ found that the degree of PWS blanching correlates well to the decrease in perfusion measured by LSCI.

Finally, as pointed out by Mennes et al., ${ }^{59}$ LSCI could be of help in the assessment of diabetic foot ulcers that are related to local ischemia. They point out that no such study has yet been performed on the diabetic foot population even though there seems to be a lot of potential based on a comparable study by others. ${ }^{60}$ Also, similar studies have been published on the use of LSCI in microcirculatory flow measurements in venous ulcers, ${ }^{61}$ prediction of venous ulcers, ${ }^{62}$ and digital ulcers in SSc patients. $^{63}$

LSCI has made it to clinical applicability within burn surgery and dermatology. Yet, some notes are necessary. PWS birthmarks are characterized by compromised perfusion and sometimes parallel with a change in color. The latter is a concern in many studies considering it influences the optical properties. One must note that a reduction in redness in PWS treatment can falsely influence the perfusion readout. Reduction in redness implies an increase of penetration depth of light since optical absorption is reduced. If a reduction of perfusion is measured, this reduction is probably underestimated, which would not pose as a problem in this case. Additionally, no studies are published on MESI. This could potentially improve measurements as a result of the ability to generate semiquantitative data.
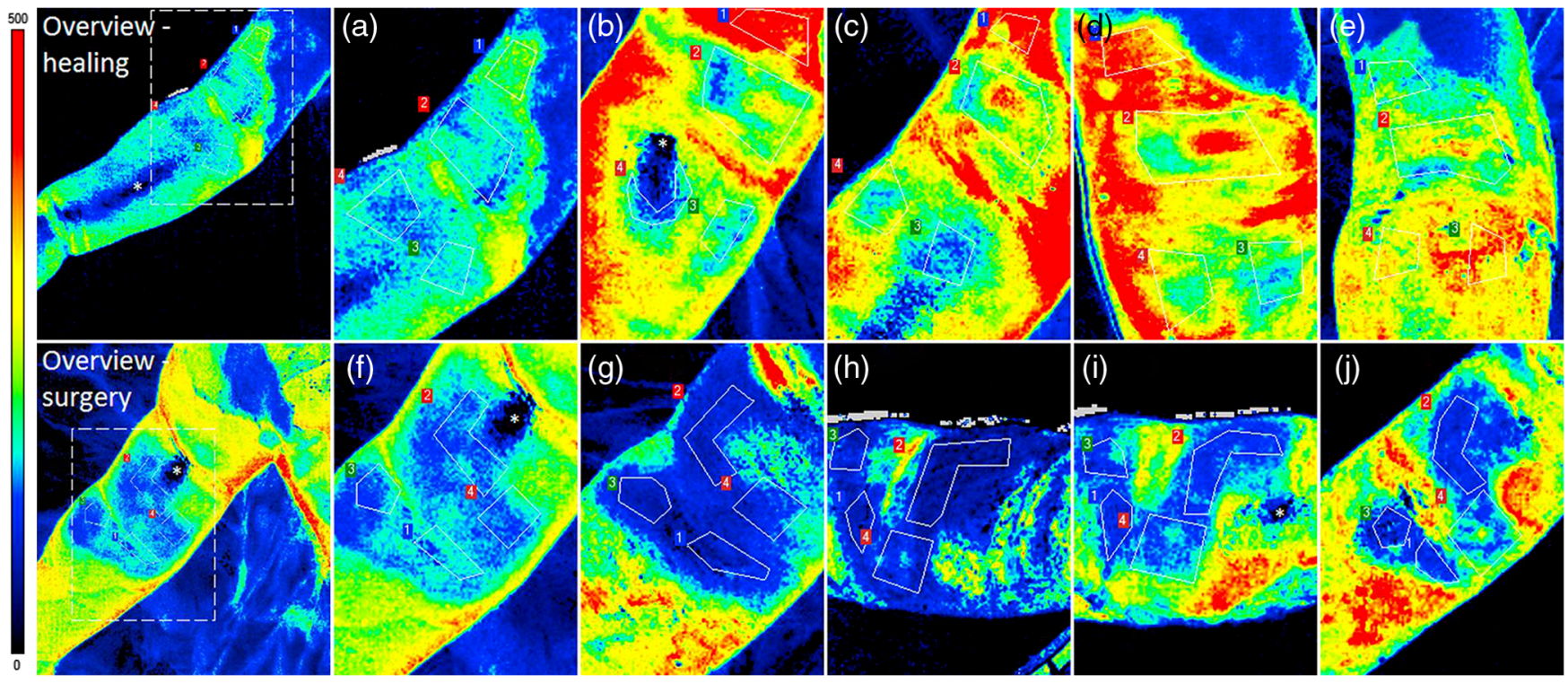

Fig. 5 Examples of perfusion in scalds with various healing times in two different children. On the upper row, a wound on the right upper and lower arm is shown that contains regions that healed between 9 and 17 days. Perfusion images (a)-(e) are acquired at (a) 14 h, (b) 4 days, (c) 6 days, (d) 8 days, and (e) 15 days after the injury. On the lower row, a wound on the upper arm of another patient is shown, which contains different regions that did not heal after 14 days and subsequently underwent surgery. Perfusion images (f)-(j) are acquired at (f) $5 \mathrm{~h}$, (g) 3 days, (h) 6 days, (i) 8 days, and (j) 10 days after the injury. The asterisks $\left(^{*}\right)$ indicates area with erroneously low perfusion values due to specular reflections. The color bar on the left side indicates the perfusion scale (0 to $500 \mathrm{PU})$. Figure reprinted from Fig. 3 in Ref. 52, () 2019, with permission from Elsevier. 

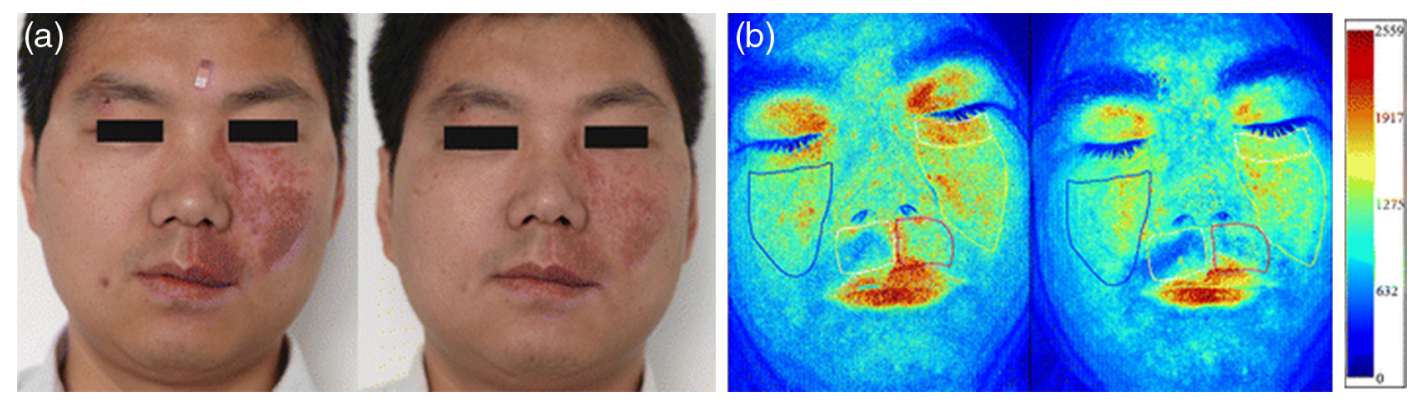

Fig. 6 Representative data of a 33-year-old male PWS patient before and at follow-up after V-PDT: (a) digital photos before and at follow-up and (b) pseudocolor LSI maps before and at follow-up. Figure reprinted from Fig. 4 in Ref. 56, (c) 2019, with permission from Springer.

\subsection{Ophthalmology}

LSCI is extensively used in ophthalmology and is currently the largest field of research. A different setup and terminology are used contrary to the sections above and beyond. A typical setup consists of a fundus camera with a laser diode and a charge coupled device image sensor. ${ }^{64}$ Depending on the application, either a laser diode (to measure deeper retinal flow) or a blue component argon laser (for superficial retinal flow) is used. Within this field, scanning times are restricted to prevent possible damage inflicted to the eye due to the use of a laser. Perfusion measurements of the choroidal circulation can be critical for the diagnosis of various diseases such as glaucoma, retinopathy, and macular degeneration. ${ }^{65}$ The retina has two separate blood supplies by virtue of the high metabolic activity. The choroidal circulation feeds the choroidal tissues and the outer section of the retina, and the retinal circulation feeds the inner portion of the retina. Tamaki et al. ${ }^{66}$ devised the first ever digital application of LSCI to measure the retinal, optical nerve head $(\mathrm{ONH})$, and choroidal blood flow dynamics. They used the technique proposed by Fercher and Briers with the addition of digital photography $y^{2,67}$ and with the additional advantage of tracking changes in blood flow in $\mathrm{ONH}$ tissue every $15 \mathrm{~s}$. This was later improved to enable real-time measurement of the ONH and the choroid of the human eye. ${ }^{68}$ Within ophthalmology, the perfusion is typically expressed in normalized blur (NB) [Eq. (4)] or square blur ratio (SBR) [Eq. (5)]. NB is the approximation for speckle contrast where the denominator is a numerical substitute for the standard deviation to save calculation time. ${ }^{66}$ The SBR has an offset variable that corrects for the offset measured at no flow and a multiplication factor $C$ based on a calibration spinning disc. Each eye and locations within the same eye have a different calibration factor and offset resulting in the inability to compare different eyes or locations: ${ }^{64}$

$$
\begin{aligned}
& \operatorname{NB}(x, y)=\frac{I_{\text {mean }}(x, y)}{1 / 98 \sum_{k=1}^{98}\left|I_{\text {mean }}(x, y)-I(x, y, k)\right|}, \\
& \operatorname{SBR}(x, y)=C \times \frac{\left\{I_{\text {mean }}(x, y)\right\}^{2}}{\sigma^{2}}-\text { offset. }
\end{aligned}
$$

This method is used to compare dynamics of the exact same location with various time intervals up to several weeks. ${ }^{69}$ The inability to make interpatient comparisons can be overcome by analyzing qualitative measures rather than quantitative, and in this case by analyzing the pulse-waveform and comparing resulting parameters such as skew, blowout score, blowout time, rising, and falling rate. ${ }^{70} \mathrm{~A}$ pulse-waveform analysis is made on a sequence of images taken with a relatively short exposure time, i.e., roughly 118 images in 4 to 5 seconds, which are then combined over multiple heartbeats (Fig. 7). Individual characteristics such as sex, early atherosclerotic changes, glaucoma severity, and systemic hemodynamic values are shown to be related to parameters that can be extracted from the pulse waveform. ${ }^{69-73}$ One can even differentiate between habitual and nonhabitual coffee drinkers based on temporal changes in ocular blood flow. ${ }^{72}$ Many studies using LSCI are available within the field of ophthalmology since this way the effect on the cardiovascular system can be studied in great detail, for example, diabetes. ${ }^{74,75}$ Despite the fact that detection of an early atherosclerotic change can be useful, it could also unintentionally negatively influence the determination of another characteristic. The comparison of interpatient qualitative measures of blood flow dynamics has not yet been reported in other fields. However, these parameters result in a broader clinical applicability ranging from ocular disease to systemic hemodynamic monitoring.

\subsection{Neurology}

The measurement of CBF during neurosurgery is crucial in verifying that blood perfusion levels are at presurgical levels and to asses postsurgical tissue viability. ${ }^{76}$ The CBF was first studied in small animals such as rodents. ${ }^{77}$ Subjects of studies are functional provocations, such as electrical stimulation, cortical spreading depressions and hypercapnia but also physiological alterations such as hyperoxia, hyperthermia, and vascular occlusions. ${ }^{78}$ The real translation from the experimental setting to clinical practice as a standard of care has yet to come, however, some studies report on the successful clinical application of LSCI. Hecht et al. ${ }^{79}$ reported on the first clinical study that included three patients undergoing extracranial-intracranial bypass procedures. Their results were promising as they measured an increase in relative baseline after the completion of the anastomosis. They were able to verify sufficient flow replacement via the bypass subsequent to occlusion. This group used a commercially available device and placed it overtop the surgically exposed area, whereas Richards et al. ${ }^{28}$ and Parthasarathy et al. $^{76}$ developed a neurosurgical operating microscope laser speckle contrast imager. Their custom setup has the advantages of having minimal interference with the surgical procedure due to a shorter setup time, having an almost 50 times greater temporal resolution and correcting for the pulsation of the brain using an ECG-based post hoc correction. These studies both included only three patients each, however, in the second study of Hecht et al., ${ }^{80}$ thirty patients undergoing direct surgical revascularization were included giving similar 


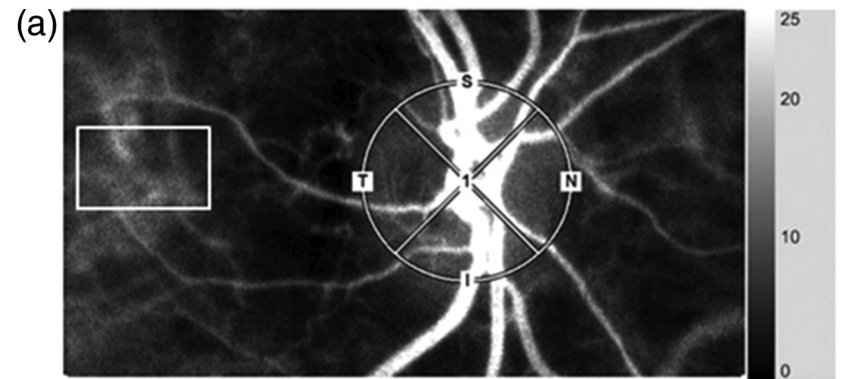

(b)
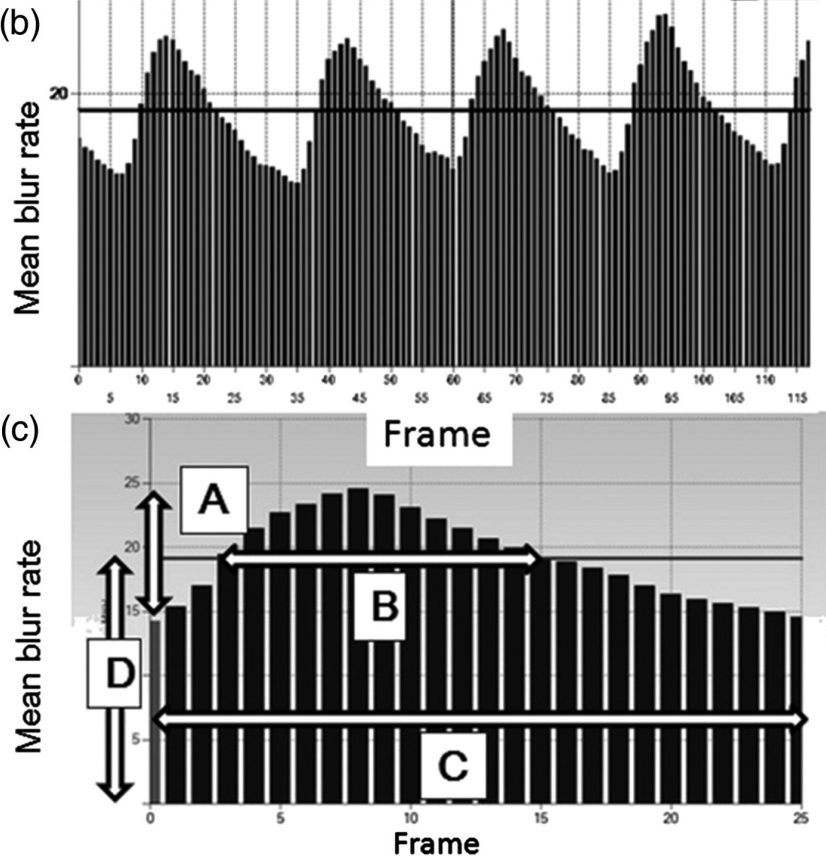

Fig. 7 The method for analyzing the mean blur rate (MBR) using laser speckle flowgraphy. (a) The gray-scale map of the total measurement area. The circle and rectangle designate the area of the $\mathrm{ONH}$ and center placed at the tissue area avoiding the retinal vessel measured. (b) The pulse waves show changes in the MBR, which is tuned to the cardiac cycle for $4 \mathrm{~s}$. The total number of frames is 118 . (c) Normalization of one pulse. MBR values are provided on this screen. a, maximal MBR-minimal MBR; $b$, the number of frames spent at one-half value of $A ; c$, the number of frames spent at one normalized pulse; $d$, the average MBR; T, temporal; $\mathrm{N}$, nasal; $\mathrm{S}$, superior; and I, inferior. Figure reprinted from Fig. 1 in Ref. 74, ( 2019, with permission from Springer. promising results concerning clinical applicability. The LSCI images might give neurosurgeons an indication of the effectiveness of the surgical revascularization as can be seen in Fig. 8. Nomura et al. ${ }^{81}$ compared ${ }^{123}$ I-iodoampethamine single-photon emission computer tomography to LSCI reporting on moderate success. Other reported uses of LSCI are the prediction of infarction, ${ }^{82}$ functional brain mapping, ${ }^{83}$ and the propagation of cortical spreading depolarization after malignant stroke. ${ }^{84}$ In the authors' opinion, LSCI is very relevant for neurosurgery. The integration in surgical practice causes minimal disturbance, especially using the microscope setup described by Parthasarathy et al. ${ }^{76}$ Future research should consist of collecting vast amounts of data on CBF assessment to determine parameters of interest based on blood flow changes, in which the surgeon can base their decision-making for further treatment. This, in combination with increased robustness, could result in general acceptance of LSCI as a standard of care within neurosurgery.

\subsection{Gastro-Intestinal Tract Surgery}

The foregoing studies are limited to exposed tissues or to an open surgical setting with some exceptions. ${ }^{42,43}$ Only limited studies on the clinical application of LSCI in the abdominal cavity are available. Eriksson et al. ${ }^{85}$ were the first to report on the in vivo application of LSCI on a visceral organ in an open surgical setting. They measured blood perfusion of the liver with occlusion of the portal vein and hepatic artery. Their conclusion is that LSCI holds promise for this clinical application although the movement artifacts are a cause of concern. Artifacts caused by the heart beating accounted for $40 \%$ of their signal and respiratory artifacts showed up as low-frequency oscillations, which seems to be a recurring theme in perfusion measurements on visceral organs. Later, others performed perfusion measurements on porcine abdominal organs using LSCI on the stomach, liver, and small intestine ${ }^{86}$ and esophagus. ${ }^{87}$ Several groups have measured microvascular blood flow during esophagectomies in humans. ${ }^{88-90}$ The first group to image the gastric microvascular perfusion was able to detect ischemic areas on gastric tube reconstructions. ${ }^{90}$ Interestingly, this study confirms that LSCI can be of help in identifying ischemic areas to reduce anastomotic leakage, which is a major complication within gastrointestinal surgery. ${ }^{91-93}$ This was later confirmed by others in Ivor-Lewis esophagectomies. $^{88}$ In a different study, LSCI was used to
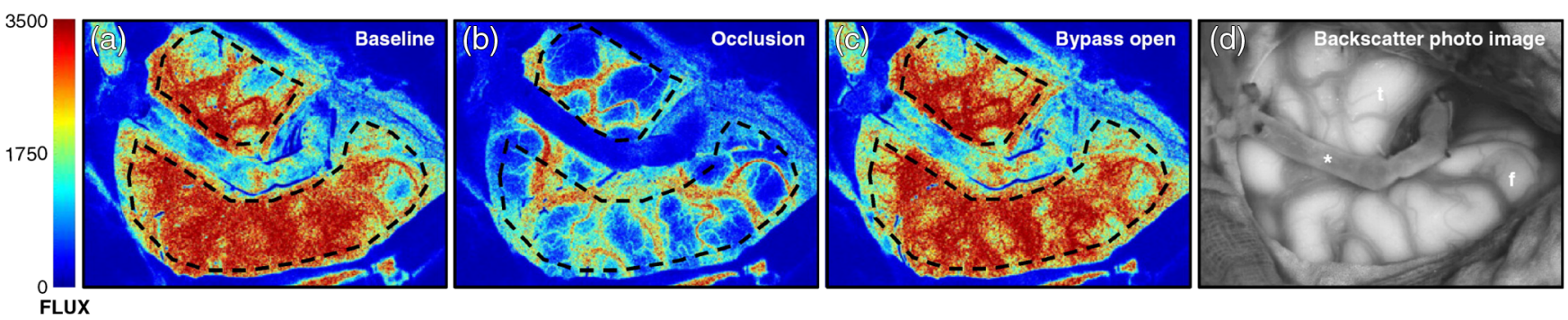

Fig. 8 Temporal response (dynamics) of LSI. LSI screenshots $(1 \mathrm{~Hz}$ ) from a continuous cortical perfusion assessment in a patient after completion of a high-flow bypass graft (saphenous vein, asterisk). Panels (a)-(c) visualize cortical perfusion (a) before, (b) during, and (c) after a test-occlusion procedure of the bypass and main aneurysm-carrying vessel with subsequent flow initiation only through the bypass graft with a high flux (red) indicating high flow and low flux (blue) indicating low flow. (d) The corresponding backscatter photo. The dashed region of interest in (a)-(c) shows the area within which the main cortical perfusion was calculated. The LSCl-specific detection of a sudden flow reduction during test occlusion and immediate reperfusion after flow initiation can be noted in the second and third panels from the left. Note the absent perfusion in the bypass graft during test occlusion (asterisk). Figure reprinted from Fig. 4(a) in Ref. 80, ( 2019, with permission from Sage Publications. 
investigate the effect of thoracic epidural anesthesia, which can provoke hypotension, ${ }^{89}$ proving that LSCI can be used to image the gastric microcirculation in real-time. Next to this, LSCI is sensitive enough to detect even the slightest changes in the gastric microvascular perfusion. Finally, Knudsun et al. ${ }^{94}$ used LSCI to measure microperfusion on the bowel of a newborn with necrotizing enterocolitis. The perfusion values matched with the macroscopic findings of the surgeon for the surgical resection lines in a post hoc analysis. Interestingly, the post hoc analysis showed another region with low perfusion values that the surgeon was not able to distinguish and did not include in the surgery. An ischemic stenosis was found during reoperation 14 days postsurgery, which suggests that in this case LSCI could have prevented reoperation. This is a very interesting note concerning the predictive value of LSCI even though this was a case study. These studies were performed in an open surgical setting, whereas very little studies are published using LSCI in an endoscopic or laparoscopic setting. Bray et al. ${ }^{43}$ was the first to use LSCI combined with an endoscope in vivo to image the blood flow in the human knee, which has been described in Sec. 3.1, after initial testing in a rabbit knee. ${ }^{42}$ Two other groups have devised an endoscope to image the retinal blood flow in rats ${ }^{95}$ and mice ${ }^{96}$ to overcome the difficulty associated with the refractive properties due to the curved cornea. Some used endoscopes, whereas Zheng et al. ${ }^{97}$ reported on the development of a multi-camera laparoscope. This was used to image intestinal vasculature in a minimal invasive setting in a swine. Our own group recently published the first-in-human application with a combination of a standard laparoscopic surgical setup with LSCI. We were able to image intestinal blood flow during a vascular occlusion test in a human. ${ }^{98}$ These are the first laparoscopic intraabdominal applications of LSCI focused on the large intestine; however, this could work for other gastro-intestinal-surgery or any other endoscopic intraabdominal application.

\subsection{Other Uses of $\mathrm{LSCl}$}

Several fields of research are highlighted in this review solely based on the authors' interests. However, other interesting applications of laser speckle are applicable in dentistry and cardiovascular research. Within dentistry and oral surgery laser speckle is used to image the gingival blood flow ${ }^{99-104}$ (Fig. 9) or the even deeper situated dental pulp flow. ${ }^{105,106}$ Another interesting study uses laser speckle to avoid the inevitable human bias in the classification of dental decay. ${ }^{107}$ They have used, as a first in literature, the $\chi^{2}$ distance histogram analysis to quantify white spot lesions rather than a traditional contrast calculation such as Eq. (1). Cardiovascular studies are largely interested in using laser speckle to assess the microvascular endothelial function of the skin under the influence of different stimuli such as transdermal iontophoretic delivery of acetylcholine ${ }^{108,109}$ or other pharmacological and physiological provocations. ${ }^{110}$ Even though LSCI is suitable for detecting altered endothelial function, Hellmann et al. ${ }^{111}$ stated that the endothelial function is altered in many cardiovascular diseases it should not function as a diagnostic nor prognostic biomarker. This is contrary to others. ${ }^{112,113}$ Also a study was published about the assessment of the forearm endothelium showing significantly impaired vasodilatory response with type I diabetes. ${ }^{114}$ Finally, dual-wavelength LSCI allows for simultaneous imaging of blood flow and the oxyhemoglobin and deoxyhemoglobin concentrations. ${ }^{115,116}$

\section{Current Limitations of LSCI}

As described above in a multitude of ways, there are still limitations that impede the clinical use of LSCI. First of all, most

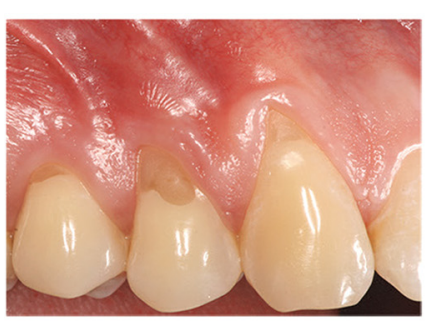

(a)

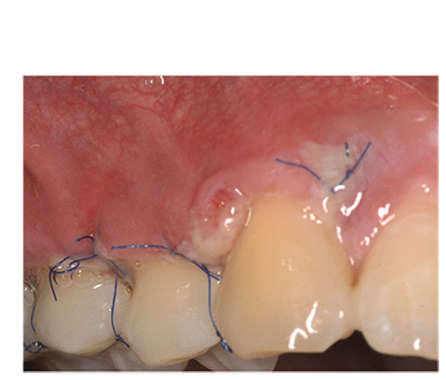

(e)

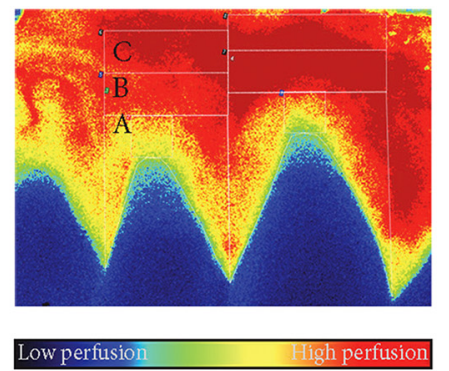

(b)

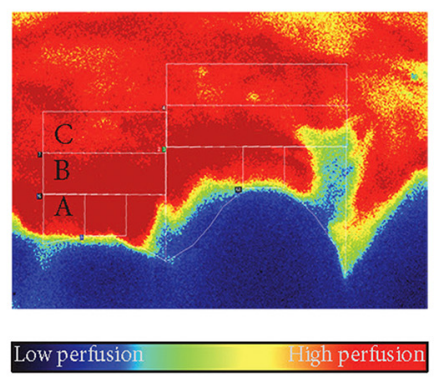

(f)

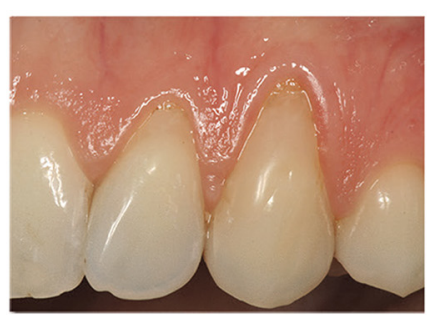

(c)

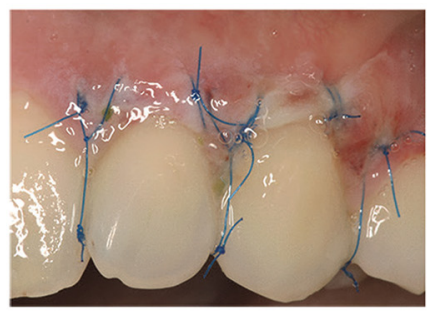

(g)

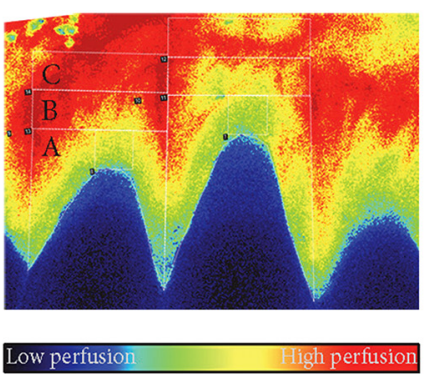

(d)

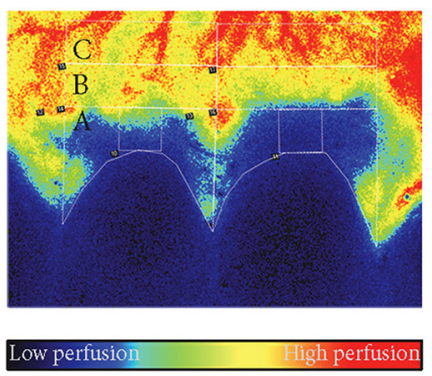

(h)

Fig. 9 Representative photographs and LSCl images of (a), (b), (e), and (f) male and (c), (d), (g), and (h) female subjects; in both cases, a combination of the modified coronally advanced tunnel and Geistlich Mucograft was used. (a)-(d) Images representing the preoperative perfusion and (e)-(h) images showing the wound healing and perfusion 3 days postoperatively. Capital letters $(A, B$, and $C)$ indicate the regions of interest for the blood flow evaluation. Figure reprinted from Fig. 1 in Ref. 100, () 2019, with permission from Hindawi. 
studies are bound to qualitative measurements and very limited interpatient comparability. Moreover, LSCI is unfortunately still not a quantitative method although great steps forward have been made by the group of Dunn et al.$^{10}$ with the introduction of multi-exposure speckle imaging. In the authors' opinion, multiple exposures should be further explored in upcoming studies. Second, the unwanted movement of tissues is the pitfall of the clinical application of laser speckle. Many studies have been published about movement artefact correction for moving tissues; however, there is no definitive all-round solution yet. ${ }^{22,28}$ This seems to be a problem for the imaging of moving tissues such as intraabdominal perfusion measurements as well as patients whom cannot remain still. Third, to get interpatient comparability these systems require either a zero-flow area within the field of view or a highly controlled environment. This zero-flow area cannot show any signs of perfusion and could be in the form of a surgical mesh or an adhesive opaque patch. A controlled environment is easily created in dermatological, ophthalmological, rheumatological, and neurological studies. In these cases, patients are either sedated or in full control of their movements. Hence, these are currently the fields with the largest clinical acceptation and application.

\section{Conclusion}

Most studies addressed in this review are clinical studies are only a fraction of all literature available on LSCI. Many studies are performed in animal models as a preclinical study, which shows great potential although they never translate into clinical applications. Currently, there are at least two commercially available laser speckle contrast imagers that share common limitations. This inherently means that most studies run into the same problem. When the above-mentioned problems are solved, with the emphasis on getting quantitative results and robust movement artefact correction, LSCI can become more than a research tool. It is a cheap, noncontact and reliable imaging modality that can measure blood perfusion at any time during a clinical examination. With the knowledge that microcirculation is an indicator for tissue viability, this technique can be applied in many more medical fields.

\section{Disclosures}

The authors declare no conflicts of interest. The authors report no funding was received for this study.

\section{Acknowledgments}

This work was supported by the knowledge and innovation fund of the Samenwerkingsverband Noord Nederland (SNN) Grant No. KEI18PR004.

\section{References}

1. A. Karliczek et al., "Surgeons lack predictive accuracy for anastomotic leakage in gastrointestinal surgery," Int. J. Colorectal Dis. 24(5), 569576 (2009).

2. A. Fercher and J. Briers, "Flow visualization by means of singleexposure speckle photography," Opt. Commun. 37(5), 326-330 (1981).

3. J. D. Briers and S. Webster, "Quasi real-time digital version of singleexposure speckle photography for full-field monitoring of velocity or flow fields," Opt. Commun. 116(1-3), 36-42 (1995).

4. J. D. Briers and S. Webster, "Laser speckle contrast analysis (LASCA): a nonscanning, full-field technique for monitoring capillary blood flow," J. Biomed. Opt. 1(2), 174-179 (1996).

5. L. M. Richards et al., "Low-cost laser speckle contrast imaging of blood flow using a webcam," Biomed. Opt. Express 4(10), 2269-2283 (2013).
6. M. Draijer et al., "Review of laser speckle contrast techniques for visualizing tissue perfusion," Lasers Med. Sci. 24(4), 639-651 (2009).

7. D. Briers et al., "Laser speckle contrast imaging: theoretical and practical limitations," J. Biomed. Opt. 18(6), 066018 (2013).

8. D. A. Boas and A. K. Dunn, "Laser speckle contrast imaging in biomedical optics," J. Biomed. Opt. 15(1), 011109 (2010).

9. L. M. Richards et al., "Intraoperative multi-exposure speckle imaging of cerebral blood flow," J. Cereb. Blood Flow Metab. 37(9), 3097-3109 (2017).

10. A. B. Parthasarathy et al., "Robust flow measurement with multiexposure speckle imaging," Opt. Express 16(3), 1975-1989 (2008).

11. R. Bonner and R. Nossal, "Model for laser Doppler measurements of blood flow in tissue," Appl. Opt. 20(12), 2097-2107 (1981).

12. J. C. Ramirez-San-Juan et al., "Simple correction factor for laser speckle imaging of flow dynamics," Opt. Lett. 39(3), 678-681 (2014).

13. D. D. Duncan and S. J. Kirkpatrick, "Can laser speckle flowmetry be made a quantitative tool?" J. Opt. Soc. Am. A 25(8), 2088-2094 (2008).

14. O. B. Thompson and M. K. Andrews, "Tissue perfusion measurements: multiple-exposure laser speckle analysis generates laser Doppler-like spectra," J. Biomed. Opt. 15(2), 027015 (2010).

15. T. Smausz, D. Zölei, and B. Hopp, "Real correlation time measurement in laser speckle contrast analysis using wide exposure time range images," Appl. Opt. 48(8), 1425-1429 (2009).

16. P. Zakharov et al., "Quantitative modeling of laser speckle imaging," Opt. Lett. 31(23), 3465-3467 (2006).

17. D. Duncan et al., "What is the proper statistical model for laser speckle flowmetry?" Proc. SPIE 6855, 685502 (2008).

18. R. Bandyopadhyay et al., "Speckle-visibility spectroscopy: a tool to study time-varying dynamics," Rev. Sci. Instrum. 76(9), 093110 (2005).

19. A. Nadort et al., "Quantitative blood flow velocity imaging using laser speckle flowmetry," Sci. Rep. 6, 25258 (2016).

20. J. Zötterman et al., "Methodological concerns with laser speckle contrast imaging in clinical evaluation of microcirculation," PLoS One 12(3), e0174703 (2017).

21. G. Mahé et al., "Impact of experimental conditions on noncontact laser recordings in microvascular studies," Microcirculation 19(8), 669-675 (2012).

22. G. Mahé et al., "Laser speckle contrast imaging accurately measures blood flow over moving skin surfaces," Microvasc. Res. 81(2), 183188 (2011).

23. S. Bahadori, T. Immins, and T. W. Wainwright, "The effect of calf neuromuscular electrical stimulation and intermittent pneumatic compression on thigh microcirculation," Microvasc. Res. 111, 37-41 (2017).

24. S. Bahadori, T. Immins, and T. W. Wainwright, "A novel approach to overcome movement artifact when using a laser speckle contrast imaging system for alternating speeds of blood microcirculation," J. vis. Exp. 126, 1-6 (2017).

25. B. Lertsakdadet et al., "Correcting for motion artifact in handheld laser speckle images," J. Biomed. Opt. 23(3), 036006 (2018).

26. L. Omarjee et al., "Optimisation of movement detection and artifact removal during laser speckle contrast imaging," Microvasc. Res. 97, 75-80 (2015).

27. G. Mahe et al., "Cutaneous microvascular functional assessment during exercise: a novel approach using laser speckle contrast imaging," Pflugers Arch. 465(4), 451-458 (2013).

28. L. M. Richards et al., "Intraoperative laser speckle contrast imaging with retrospective motion correction for quantitative assessment of cerebral blood flow," Neurophotonics 1(1), 015006 (2014).

29. P. Miao et al., "High resolution cerebral blood flow imaging by registered laser speckle contrast analysis," IEEE Trans. Biomed. Eng. 57(5), 1152-1157 (2010).

30. A. Della Rossa et al., "Alteration of microcirculation is a hallmark of very early systemic sclerosis patients: a laser speckle contrast analysis," Clin. Exp. Rheumatol. 31(2 Suppl 76), 109-114 (2013).

31. F. Gaillard-Bigot et al., "Abnormal amplitude and kinetics of digital postocclusive reactive hyperemia in systemic sclerosis," Microvasc. Res. 94, 90-95 (2014).

32. B. Ruaro et al., "Laser speckle contrast analysis: a new method to evaluate peripheral blood perfusion in systemic sclerosis patients," Ann. Rheum. Dis. 73(6), 1181-1185 (2014).

33. E. Rosato et al., "Skin perfusion of fingers shows a negative correlation with capillaroscopic damage in patients with systemic sclerosis," J. Rheumatol. 40(1), 98-99 (2013). 
34. M. Cutolo et al., "Nailfold videocapillaroscopy assessment of microvascular damage in systemic sclerosis," J. Rheumatol. 27(1), 155-160 (2000).

35. M. Cutolo et al., "Peripheral blood perfusion correlates with microvascular abnormalities in systemic sclerosis: a laser-Doppler and nailfold videocapillaroscopy study," J. Rheumatol. 37(6), 1174-1180 (2010).

36. E. Rosato et al., "Laser Doppler perfusion imaging is useful in the study of Raynaud's phenomenon and improves the capillaroscopic diagnosis," J. Rheumatol. 36(10), 2257-2263 (2009).

37. J. D. Pauling et al., "Use of laser speckle contrast imaging to assess digital microvascular function in primary Raynaud phenomenon and systemic sclerosis: a comparison using the Raynaud condition score diary," J. Rheumatol. 42(7), 1163-1168 (2015).

38. J. D. Wilkinson et al., "A multicenter study of the validity and reliability of responses to hand cold challenge as measured by laser speckle contrast imaging and thermography: outcome measures for systemic sclerosis-related Raynaud's phenomenon," Arthritis Rheumatol. 70(6), 903-911 (2018).

39. M. Cutolo et al., "Is laser speckle contrast analysis (LASCA) the new kid on the block in systemic sclerosis? A systematic literature review and pilot study to evaluate reliability of LASCA to measure peripheral blood perfusion in scleroderma patients," Autoimmun. Rev. 17(8), 775-780 (2018).

40. T. Son et al., "Continuous monitoring of arthritis in animal models using optical imaging modalities," J. Biomed. Opt. 19(10), 106010 (2014).

41. J. Dunn et al., "A transmissive laser speckle imaging technique for measuring deep tissue blood flow: An example application in finger joints," Lasers Surg. Med. 43(1), 21-28 (2011).

42. K. R. Forrester et al., "Endoscopic laser imaging of tissue perfusion: new instrumentation and technique," Lasers Surg. Med. 33(3), 151157 (2003).

43. R. C. Bray et al., "Endoscopic laser speckle imaging of tissue blood flow: applications in the human knee," J. Orthop. Res. 24(8), 1650 1659 (2006).

44. S. A. Pape, C. A. Skouras, and P. O. Byrne, "An audit of the use of laser Doppler imaging (LDI) in the assessment of burns of intermediate depth," Burns 27(3), 233-239 (2001).

45. D. P. Orgill, "Excision and skin grafting of thermal burns," N. Engl. J. Med. 360(9), 893-901 (2009).

46. M. Kaiser et al., "Noninvasive assessment of burn wound severity using optical technology: a review of current and future modalities," Burns 37(3), 377-386 (2011).

47. D. J. McGill et al., "Assessment of burn depth: a prospective, blinded comparison of laser Doppler imaging and videomicroscopy," Burns 33(7), 833-842 (2007).

48. C. J. Stewart et al., "A comparison of two laser-based methods for determination of burn scar perfusion: laser Doppler versus laser speckle imaging," Burns 31(6), 744-752 (2005).

49. C. Crouzet et al., "Acute discrimination between superficial-partial and deep-partial thickness burns in a preclinical model with laser speckle imaging," Burns 41(5), 1058-1063 (2015).

50. F. Lindahl, E. Tesselaar, and F. Sjöberg, "Assessing paediatric scald injuries using laser speckle contrast imaging," Burns 39(4), 662-666 (2013).

51. R. Mirdell et al., "Accuracy of laser speckle contrast imaging in the assessment of pediatric scald wounds," Burns 44(1), 90-98 (2018).

52. R. Mirdell et al., "Microvascular blood flow in scalds in children and its relation to duration of wound healing: a study using laser speckle contrast imaging," Burns 42(3), 648-654 (2016).

53. S. A. Sharif et al., "Noninvasive clinical assessment of port-wine stain birthmarks using current and future optical imaging technology: a review," Br. J. Dermatol. 167(6), 1215-1223 (2012).

54. Y. C. Huang et al., "Blood flow dynamics after laser therapy of port wine stain birthmarks," Lasers Surg. Med. 41(8), 563-571 (2009).

55. Y. C. Huang et al., "Noninvasive blood flow imaging for real-time feedback during laser therapy of port wine stain birthmarks," Lasers Surg. Med. 40(3), 167-173 (2008).

56. J. Ren et al., "Assessment of tissue perfusion changes in port wine stains after vascular targeted photodynamic therapy: a short-term follow-up study," Lasers Med. Sci. 29(2), 781-788 (2014).
57. H. Qiu et al., "Monitoring microcirculation changes in port wine stains during vascular targeted photodynamic therapy by laser speckle imaging," Photochem. Photobiol. 88(4), 978-984 (2012).

58. B. Yang et al., "Intraoperative, real-time monitoring of blood flow dynamics associated with laser surgery of port wine stain birthmarks," Lasers Surg. Med. 47(6), 469-475 (2015).

59. O. A. Mennes et al., "Novel optical techniques for imaging microcirculation in the diabetic foot," Curr. Pharm. Des. 24(12), 1304-1316 (2018).

60. M. Hellmann et al., "Cutaneous iontophoresis of treprostinil, a prostacyclin analog, increases microvascular blood flux in diabetic malleolus area," Eur. J. Pharmacol. 758, 123-128 (2015).

61. L. C. Huisman, C. den Bakker, and C. H. A. Wittens, "Microcirculatory changes in venous disease," Phlebology 28(Suppl 1), 73-78 (2013).

62. T. M. van Vuuren et al., "Prediction of venous wound healing with laser speckle imaging," Phlebology 32(10), 658-664 (2017).

63. B. Ruaro et al., "Short-term follow-up of digital ulcers by laser speckle contrast analysis in systemic sclerosis patients," Microvasc. Res. 101, 82-85 (2015).

64. T. Sugiyama et al., "Use of laser speckle flowgraphy in ocular blood flow research," Acta Ophthalmol. (Copenh) 88(7), 723-729 (2010).

65. X. Wei et al., "Assessment of flow dynamics in retinal and choroidal microcirculation," Surv. Ophthalmol. 63(5), 646-664 (2018).

66. Y. Tamaki et al., "Noncontact, two-dimensional measurement of retinal microcirculation using laser speckle phenomenon," Invest. Ophthalmol. Vis. Sci. 35(11), 3825-3834 (1994).

67. J. D. Briers and A. F. Fercher, "Retinal blood-flow visualization by means of laser speckle photography," Invest. Ophthalmol. Vis. Sci. 22(2), 255-259 (1982).

68. Y. Tamaki et al., "Real-time measurement of human optic nerve head and choroid circulation, using the laser speckle phenomenon," Jpn. J. Ophthalmol. 41(1), 49-54 (1997).

69. H. Kunikata and T. Nakazawa, "Recent clinical applications of laser speckle flowgraphy in eyes with retinal disease," Asia-Pacific $J$. Ophthalmol. 5(2), 151-158 (2016).

70. S. Tsuda et al., "Pulse-waveform analysis of normal population using laser speckle flowgraphy," Curr. Eye Res. 39(12), 1207-1215 (2014).

71. T. Shiba et al., "Optic nerve head circulation determined by pulse wave analysis is significantly correlated with cardio ankle vascular index, left ventricular diastolic function, and age," J. Atheroscler. Thromb. 19(11), 999-1005 (2012).

72. A. Ismail et al., "Pulse waveform analysis on temporal changes in ocular blood flow due to caffeine intake: a comparative study between habitual and non-habitual groups," Graefe's Arch. Clin. Exp. Ophthalmol. 256(9), 1711-1721 (2018).

73. T. Shiba et al., "Pulse-wave analysis of optic nerve head circulation is significantly correlated with brachial-ankle pulse-wave velocity, carotid intima-media thickness, and age," Graefe's Arch. Clin. Exp. Ophthalmol. 250(9), 1275-1281 (2012).

74. C. Shiba et al., "Relationship between glycosylated hemoglobin A1c and ocular circulation by laser speckle flowgraphy in patients with/ without diabetes mellitus," Graefes Arch. Clin. Exp. Ophthalmol. 254(9), 1801-1809 (2016).

75. K. Hashimoto et al., "The relationship between advanced glycation end products and ocular circulation in type 2 diabetes," J. Diabetes Complications 30(7), 1371-1377 (2016).

76. A. B. Parthasarathy et al., "Laser speckle contrast imaging of cerebral blood flow in humans during neurosurgery: a pilot clinical study," J. Biomed. Opt. 15(6), 066030 (2010).

77. A. K. Dunn et al., "Dynamic imaging of cerebral blood flow using laser speckle," J. Cereb. Blood Flow Metab. 21(3), 195-201 (2001).

78. S. M. S. Kazmi et al., "Expanding applications, accuracy, and interpretation of laser speckle contrast imaging of cerebral blood flow," J. Cereb. Blood Flow Metab. 35(7), 1076-1084 (2015).

79. N. Hecht et al., "Intraoperative monitoring of cerebral blood flow by laser speckle contrast analysis," Neurosurg. Focus 27(4), E11 (2009).

80. N. Hecht et al., "Laser speckle imaging allows real-time intraoperative blood flow assessment during neurosurgical procedures," J. Cereb. Blood Flow Metab. 33(7), 1000-1007 (2013).

81. S. Nomura et al., "Reliability of laser speckle flow imaging for intraoperative monitoring of cerebral blood flow during cerebrovascular 
surgery: comparison with cerebral blood flow measurement by single photon emission computed tomography," World Neurosurg. 82(6), e753-e757 (2014).

82. N. Hecht et al., "Infarct prediction by intraoperative laser speckle imaging in patients with malignant hemispheric stroke," J. Cereb. Blood Flow Metab. 36(6), 1022-1032 (2016).

83. E. Klijn et al., "Laser speckle imaging identification of increases in cortical microcirculatory blood flow induced by motor activity during awake craniotomy," J. Neurosurg. 118(2), 280-286 (2013).

84. J. Woitzik et al., "Propagation of cortical spreading depolarization in the human cortex after malignant stroke," Neurology 80(12), 10951102 (2013).

85. S. Eriksson et al., "Laser speckle contrast imaging for intraoperative assessment of liver microcirculation: a clinical pilot study," Med. Devices (Auckland, N.Z.) 7, 257-261 (2014).

86. R. Ambrus et al., "Laser speckle contrast imaging for monitoring changes in microvascular blood flow," Eur. Surg. Res. 56(3-4), 87-96 (2016).

87. E. Klijn et al., "The effect of perfusion pressure on gastric tissue blood flow in an experimental gastric tube model," Anesth. Analg. 110(2), 541-546 (2010).

88. R. Ambrus et al., "A reduced gastric corpus microvascular blood flow during Ivor-Lewis esophagectomy detected by laser speckle contrast imaging technique," Scand. J. Gastroenterol. 52(4), 455-461 (2017).

89. R. Ambrus et al., "Evaluation of gastric microcirculation by laser speckle contrast imaging during esophagectomy," J. Am. Coll. Surg. 225(3), 395-402 (2017).

90. D. M. J. Milstein et al., "Laser speckle contrast imaging identifies ischemic areas on gastric tube reconstructions following esophagectomy," Medicine (Baltimore) 95(25), e3875 (2016).

91. A. Karliczek et al., "Intraoperative assessment of microperfusion with visible light spectroscopy in esophageal and colorectal anastomoses," Eur. Surg. Res. 41(3), 303-311 (2008).

92. E. F. Midura et al., "Risk factors and consequences of anastomotic leak after colectomy: a national analysis," Dis. Colon Rectum 58(3), 333-338 (2015).

93. J. Bruce et al., "Systematic review of the definition and measurement of anastomotic leak after gastrointestinal surgery," Br. J. Surg. 88(9), 1157-1168 (2001).

94. K. B. K. Knudsen et al., "Laser speckle contrast imaging to evaluate bowel lesions in neonates with NEC," Eur. J. Pediatr. Surg. Rep. 5(1), e43-e46 (2017).

95. A. Ponticorvo et al., "Laser speckle contrast imaging of blood flow in rat retinas using an endoscope," J. Biomed. Opt. 18(9), 090501 (2013).

96. T. H. Kong et al., "Monitoring blood-flow in the mouse cochlea using an endoscopic laser speckle contrast imaging system," PloS One 13(2), e0191978 (2018).

97. C. Zheng, L. Wai Lau, and J. Cha, "Dual-display laparoscopic laser speckle contrast imaging for real-time surgical assistance," Biomed. Opt. Express 9(12), 5962-5981 (2018).

98. W. Heeman et al., "Application of laser speckle contrast imaging in laparoscopic surgery," Biomed. Opt. Express 10(4), 2010-2010 (2019).

99. C. Regan et al., "Design and evaluation of a miniature laser speckle imaging device to assess gingival health,” J. Biomed. Opt. 21(10), 104002 (2016).

100. E. Molnár et al., "Evaluation of laser speckle contrast imaging for the assessment of oral mucosal blood flow following periodontal plastic surgery: an exploratory study," Biomed Res. Int. 2017, 1-11 (2017).

101. R. Fazekas et al., "Functional characterization of collaterals in the human gingiva by laser speckle contrast imaging," Microcirculation 25(3), e12446 (2018).

102. E. Molnár et al., "Assessment of the test-retest reliability of human gingival blood flow measurements by laser speckle contrast imaging in a healthy cohort," Microcirculation 25(2), e12420 (2018).

103. T. Nakamoto et al., "Two-dimensional real-time blood flow and temperature of soft tissue around maxillary anterior implants," Implant Dent. 21(6), 522-527 (2012).

104. N. Kajiwara et al., "Soft tissue biological response to zirconia and metal implant abutments compared with natural tooth: microcirculation monitoring as a novel bioindicator," Implant Dent. 24(1), 37-41 (2015).
105. C. Regan et al., "Fiber-based laser speckle imaging for the detection of pulsatile flow," Lasers Surg. Med. 47(6), 520-525 (2015).

106. S. K. Dick et al., "Characterization of blood flow rate in dental pulp by speckle patterns of backscattered light from an in vivo tooth," J. Biomed. Opt. 19(10), 106012 (2014).

107. L. G. Gavinho et al., "Detection of white spot lesions by segmenting laser speckle images using computer vision methods," Lasers Med. Sci. 33(7), 1565-1571 (2018).

108. I. Cordovil et al., "Evaluation of systemic microvascular endothelial function using laser speckle contrast imaging," Microvasc. Res. 83(3), 376-379 (2012).

109. J. P. Borges et al., "The impact of exercise frequency upon microvascular endothelium function and oxidative stress among patients with coronary artery disease," Clin. Physiol. Funct. Imaging 38(5), 840-846 (2018).

110. F. Iredahl et al., "Non-invasive measurement of skin microvascular response during pharmacological and physiological provocations," PloS One 10(8), e0133760 (2015).

111. M. Hellmann, M. Roustit, and J. L. Cracowski, "Skin microvascular endothelial function as a biomarker in cardiovascular diseases?" Pharmacol. Rep. 67(4), 803-810 (2015).

112. E. G. Souza et al., "Impairment of systemic microvascular endothelial and smooth muscle function in individuals with early-onset coronary artery disease: studies with laser speckle contrast imaging," Coron. Artery Dis. 25(1), 23-28 (2014).

113. J. P. Borges et al., "A novel effective method for the assessment of microvascular function in male patients with coronary artery disease: a pilot study using laser speckle contrast imaging," Braz. J. Med. Biol. Res. 49(10), e5541 (2016).

114. A. S. Matheus et al., "Assessment of microvascular endothelial function in type 1 diabetes using laser speckle contrast imaging," J. Diabetes Complications 31(4), 753-757 (2017).

115. J. Qin et al., "Hemodynamic and morphological vasculature response to a burn monitored using a combined dual-wavelength laser speckle and optical microangiography imaging system," Biomed. Opt. Express 3(3), 455-466 (2012).

116. J. Wang et al., "Dual-wavelength laser speckle imaging to simultaneously access blood flow, blood volume, and oxygenation using a color CCD camera," Opt. Lett. 38(18), 3690-3692 (2013).

Wido Heeman received his master's degree in biomedical engineering from the University of Groningen in 2018. He is working at LIMIS development and is currently appointed as a PhD student in biomedical engineering at the Faculty Campus Fryslân, University of Groningen, The Netherlands. His ongoing research includes the development of a more robust laser speckle contrast imaging tool for image tissue perfusion.

Wiendelt Steenbergen received his master's degree in aerospace engineering from Delft University of Technology in 1988 and his PhD in fluid dynamics from the Eindhoven University of Technology in 1995. In 2010, he became a full professor and group leader of the Biomedical Photonic Imaging Group, University of Twente, Enschede, The Netherlands. His current research interests are speckle-based perfusion imaging, combined photoacoustic and ultrasound imaging, and quantification of photoacoustic imaging using acousto-optics.

Gooitzen M. van Dam started working as a surgeon in 1995 at the University Medical Centre Groningen. In 2002, he installed the BioOptical Imaging Centre Groningen containing several imaging instruments for in vivo imaging. Currently, he is a head of the Optical Molecular Imaging Groningen group focused on the clinical translation and development of new optical tracers and instrumentation into the clinic in various fields of medicine using optical/optoacoustic imaging instruments in combination with targeted tracers.

E. Christiaan Boerma received his $\mathrm{PhD}$ in medicine, with a thesis on microcirculatory alterations during sepsis, from the University of Amsterdam in 2009. He was registered as medical doctor (MD) in 1990 at the Vrije Universiteit Amsterdam. Since 2002, he has been a consultant at Intensive Care Medicine, Medical Centre Leeuwarden, The Netherlands. His current research includes macro- and microcirculatory derangements in the critically ill, speckle-based perfusion imaging, and medicine-based evidence. 